\title{
262 PRECLINICAL CHARACTERIZATION OF HUMANIZED ANTI-SIGLEC-15 ANTIBODIES THAT RESCUE T CELLS FROM MACROPHAGE-MEDIATED IMMUNE SUPPRESSION
}

Huyen Dinh, Sam Lam, Valerie Wall, Francisco Zapata, Darbie Whitman, Ramya Chandrasekaran, Lauren Loh, Texia Loh, Tom Graddis, Peter Probst*, Myriam Bouchlaka. OncResponse Inc., Seattle, WA, USA

Background Siglec-15 is an immunosuppressive sialic acid-binding Ig-like lectin expressed by myeloid cells, tumor associated macrophages (TAMs), and some human tumors. Interactions between Siglec-15 on TAMs and sialoglycans found on cancer cells contribute to the immunosuppressive tumor microenvironment. Furthermore, Siglec-15 expressed by TAMs inhibits anti-tumor immune responses by engaging unknown immune checkpoint(s) on $\mathrm{T}$ cells. Notably, the mutually exclusive expression of Siglec-15 and the checkpoint ligand PD-L1 in the tumor tissue emphasizes Siglec-15 as an attractive target for combination immunotherapy. Anti-Siglec-15 antibody is currently being evaluated in clinical trials for the treatment of cancer.

Methods Anti-Siglec-15 antibodies were cloned from B cells derived from rabbits immunized with human Siglec-15 protein. The antibodies were evaluated for binding to human and cynomolgus Siglec-15 by enzyme-linked immunosorbent assay (ELISA). Top clones were selected based on activity in a panel of functional and phenotypic assays using primary human macrophages and $\mathrm{T}$ cells and were subsequently fully humanized. The humanized anti-Siglec-15 IgG1 antibodies were screened for binding to human and cynomolgus Siglec-15 by ELISA, binding to cells expressing Siglec-15, and ability to rescue $\mathrm{T}$ cell functional activity (proliferation and IFN- $\gamma$ ) from M2c-mediated immune suppression in vitro. The pharmacokinetics of lead humanized Siglec-15 IgG1 antibodies and their anti-tumor activity were evaluated in humanized mouse models.

Results We have identified a panel of fully humanized antiSiglec-15 antibodies that bind to recombinant human and cynomolgus Siglec-15 proteins, to Siglec-15-expressing cell lines and immunosuppressive M2c macrophages without appreciable binding to other Siglec family members. Lead antibodies were identified using functional screens modeling Siglec-15-mediated immune suppression by M2c macrophages. These antibodies restored $\mathrm{T}$ cell immune responses in two different M2c/CD8 $\mathrm{T}$ cell coculture assays. In the first model, lead antibodies rescued the proliferative and IFN- $\gamma$ responses of anti-CD3-activated human $\mathrm{T}$ cells from the inhibitory activity of $\mathrm{M} 2 \mathrm{c}$ macrophages. In the second model, these antibodies restored the ability of exhausted CD8 T cells to secrete IFN- $\gamma$ in the presence of M2c macrophages. Lead antibodies demonstrated a half-life of 6-11 days in humanized FcRn mice, and tumor growth inhibition in humanized NSG-SGM3 mice.

Conclusions We have identified novel humanized anti-Siglec-15 antibodies that restore effector function of activated and exhausted $\mathrm{T}$ cells from M2c-mediated immune suppression, with excellent half-life and anti-tumor activity in humanized mouse models. These data provide a strong rationale for further development of these antibodies for anti-cancer immunotherapy.

http://dx.doi.org/10.1136/jitc-2021-SITC2021.262 